\title{
A New Generalized Odd Gamma Uniform Distribution: Mathematical Properties, Application and Simulation
}

\author{
B. HOSSIENI, M. AFSHARI, M. ALIZADEH, H. KARAMIKABIR \\ Department of Statistics, Faculty of Intelligent Systems Engineering and Data Science \\ Persian Gulf University \\ Bushehr, IRAN
}

Abstract: - In many applied areas there is a clear need for the extended forms of the well-known distributions. The
new distributions are more flexible to model real data that present a high degree of skewness and kurtosis, such
that each one solves a particular part of the classical distribution problems. In this paper, a new two-parameter
Generalized Odd Gamma distribution, called the (GOGaU) distribution, is introduced and the fitness capability of
this model are investigated. Some structural properties of the new distribution are obtained. The different meth-
ods including: Maximum likelihood estimators, Bayesian estimators (posterior mean and maximum a posterior),
least squares estimators, weighted least squares estimators, Cramér-von-Mises estimators, Anderson-Darling and
right tailed Anderson-Darling estimators are discussed to estimate the model parameters. In order to perform the
applications, the importance and flexibility of the new model are also illustrated empirically by means of two real
data sets. For simulation Stan and JAGS software were utilized in which we have built the GOGaU JAGS modul

Key-Words: Generalized odd gamma-G; Maximum likelihood; Bayes; Stan; JAGS software; Moment; Entropy

Received: February 5, 2021. Revised: July 9, 2021. Accepted: July 21, 2021. Published: July 31, 2021.

\section{Introduction}

The statistical analysis and modeling of data sets are essential in almost all applied sciences including, biomedical science, engineering, finance, agriculture and insurance, amongst others.

Even though the new distributions sometimes include complicated mathematical formulations, each of them is able to model particular state of real data properly. Since the new processors computers are readily able to accomplish the accurate and fast numerical computations, there is no concern for good estimation of parameters, and this achievement has presented a remarkable help to this field of statistics.

Based on T-X idea by Alzaatreh et al. [2], GOGa$\mathrm{G}$ is introduced by Hosseini et al. [12] with cumulative distribution function(cdf) given by

$$
\begin{aligned}
F(x ; \alpha, \beta, \boldsymbol{\xi}) & =\int_{0}^{\frac{G\left(x ; \boldsymbol{\xi} \boldsymbol{\xi}^{\beta}\right.}{1-G(x ; \boldsymbol{\xi})^{\beta}}} \frac{t^{\alpha-1} e^{-t}}{\Gamma(\alpha)} d t \\
& =\frac{\gamma\left(\alpha, \frac{G(x ; \boldsymbol{\xi})^{\beta}}{1-G(x ; \boldsymbol{\xi})^{\beta}}\right)}{\Gamma(\alpha)} \quad x \in R .(1)
\end{aligned}
$$

where $\alpha, \beta>0$ are two additional shape parameters, $\boldsymbol{\xi}$ is the parameter for baseline $\operatorname{cdf} G$ and $\gamma(\alpha, x)=$ $\int_{0}^{x} t^{\alpha-1} e^{-t} d t$ denote the incomplete gamma function. If $G$ is standard uniform $(\mathrm{U}(0,1))$, and $X$ is a random variable such that $X \sim G O G a U(\alpha, \beta)$ then following probability density function (pdf) and hazard function (hrf) of GOGaU are obtained as follows:

$$
f(x ; \alpha, \beta)=\frac{\beta x^{\alpha \beta-1} e^{\frac{-x^{\beta}}{1-x^{\beta}}}}{\Gamma(\alpha)\left(1-x^{\beta}\right)^{\alpha+1}}, \quad 0 \leq x \leq
$$

and

$$
\begin{aligned}
& \tau(x ; \alpha, \beta)= \frac{\beta x^{\alpha \beta-1} e^{\frac{-x^{\beta}}{1-x^{\beta}}}}{\left(1-x^{\beta}\right)^{\alpha+1}\left[\Gamma(\alpha)-\gamma\left(\alpha, \frac{x^{\beta}}{1-x^{\beta}}\right)\right]}, \\
& 0 \leq x \leq 1 .
\end{aligned}
$$

As it is shown in Figure 11, the curves with different states of density function including symmetric density function (approximately), mild and high skewed (right and left) are produced. In Figure 8 one can see some curves of the hazard function of the GOGaU distribution for some parameters. Moreover, the $\mathrm{U}$ shape and increasing hazard functions are producible by GOGaU.

Some relations between the GOGaU family and gamma distribution are provided by the following proposition:

Proposition 1.1. Let $X \sim G O G a U(\alpha, \beta)$ and $Y=$ $\frac{X^{\beta}}{1-X^{\beta}}$, then $Y \sim \Gamma(\alpha, 1)$.

The rest of this paper is presented in different sections as follows:

In Section 2, we discuss the distributional properties of the proposed distribution, including quantile functions, asymptotes, expansions for pdf and 

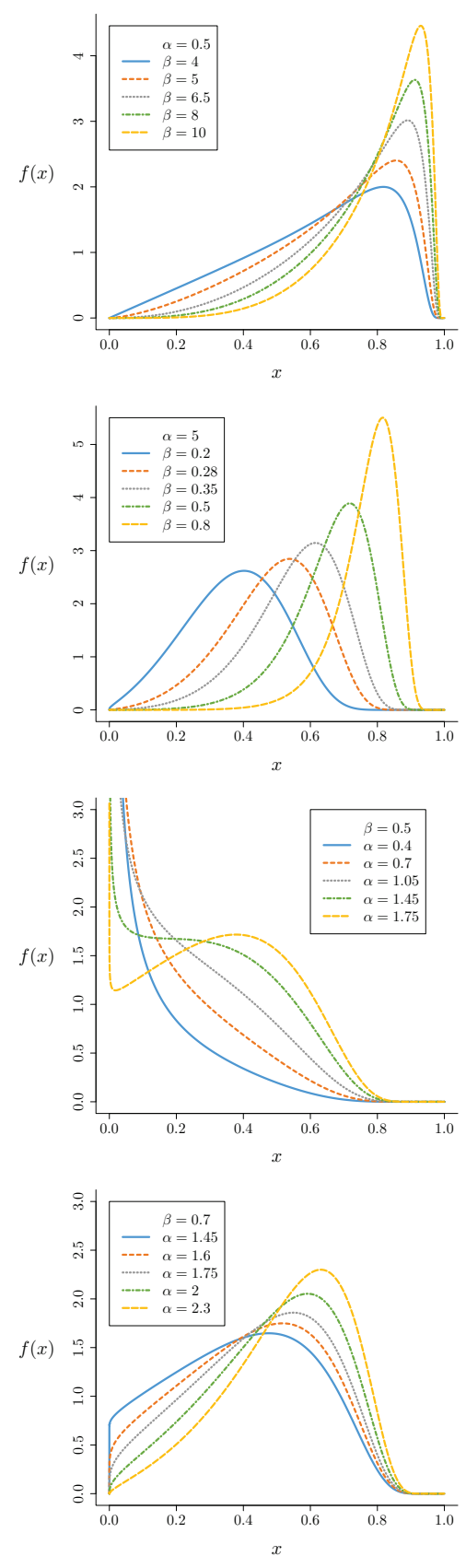

Figure 1: The sample curves of density function of GOGU01.

cdf, moments and antropy. In Section 3, parameters are estimated by Maximum likelihood, Bayesian (posterior mean and maximum a posterior), least squares, weighted least squares, Cramér-von-Mises, Anderson-Darling and right tailed Anderson-Darling methods. In Section 4, a simulation study is conducted to compare the performance of estimators. For this section, the GOGaU JAGS module is built. In Section 5, Applications with real data sets and compared to other famous well known competing are con- sidered. Finally, Section 6 offers some concluding remarks.
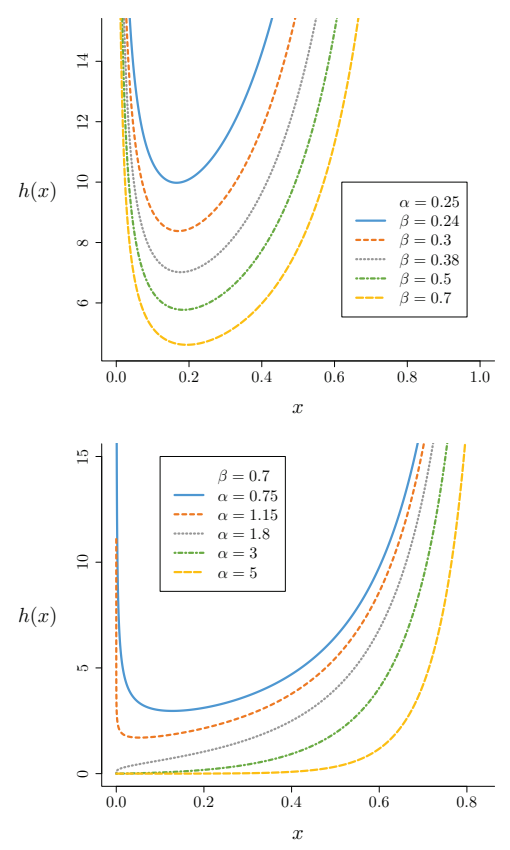

Figure 2: The sample curves of hazard function of GOGU01.

\section{Statistical properties}

In this section Some statistical properties of the new model such as Quantile function, limit behavior, moments, moment generating function, entropy are derived.

\subsection{Quantile function}

Considering (1) quantile function (qf) $X$ is obtained as follows: If $V \sim \Gamma(\alpha, 1)$ then the solution of nonlinear equation $x_{v}=\left(\frac{V}{1+V}\right)^{\frac{1}{\beta}}$ has cdf (11).

\subsection{Limit behavior}

Proposition 2.1. The asymptotic of equation (I), (2) and (3) when $x \rightarrow 0$ are given by

$$
\begin{aligned}
& F(x) \sim \frac{x^{\alpha \beta}}{\alpha \Gamma(\alpha)} \\
& f(x) \sim \frac{\beta x^{\alpha \beta-1}}{\Gamma(\alpha)} \\
& \tau(x) \sim \frac{\beta x^{\alpha \beta-1}}{\Gamma(\alpha)}
\end{aligned}
$$


Proposition 2.2. The asymptotic of equation (17), (2) and (3) when $x \rightarrow 1$ are given by

$$
\begin{gathered}
\bar{F}(x) \sim 1-\frac{\gamma\left(\alpha, \frac{1}{\beta(1-x)}\right)}{\Gamma(\alpha)} \\
f(x) \sim \frac{e^{\frac{-1}{\beta(1-x)}}}{\beta^{\alpha} \Gamma(\alpha)(1-x)^{\alpha+1}} \\
\tau(x) \sim \frac{g(x) e^{\frac{-1}{\beta(1-x)}}}{\beta^{\alpha}\left[\Gamma(\alpha)-\gamma\left(\alpha, \frac{1}{\beta(1-x)}\right)\right](1-x)^{\alpha+1}}
\end{gathered}
$$

We can evaluate the effect of the parameters on the tails of the distribution using the above equations.

\subsection{Expansion for Pdf and Cdf and hrf}

Using generalized binomial and Taylor expansion one can obtain the following equation:

$$
\begin{aligned}
f(x) & =\frac{\beta x^{\alpha \beta-1}}{\Gamma(\alpha)\left(1-x^{\beta}\right)^{\alpha+1}} \sum_{i=0}^{\infty} \frac{(-1)^{i}}{i !}\left(\frac{x^{\beta}}{1-x^{\beta}}\right)^{i} \\
& =\frac{\beta}{\Gamma(\alpha)} \sum_{i=0}^{\infty} \sum_{j=0}^{\infty} \frac{(-1)^{i}}{i !}\left(\begin{array}{c}
-\alpha-i-1 \\
j
\end{array}\right) x^{\beta(\alpha+i+j)-1} \\
& =\sum_{i=0}^{\infty} \sum_{j=0}^{\infty} w_{i, j} h_{\beta(\alpha+i+j)}(x)
\end{aligned}
$$

where $w_{i, j}=\frac{(-1)^{i}\left(\begin{array}{c}-\alpha-1 \\ j\end{array}\right)}{i ![\alpha+i+j] \Gamma(\alpha)}$ and $h_{\beta}(x)=\beta x^{\beta-1}$, denote the pdf of Beta distribution with parameters $\beta$ and 1.

\subsection{Moments}

The $r$ th ordinary moment of $X$ is given by follows:

$$
\begin{aligned}
\mu_{r}^{\prime} & =E\left(X^{r}\right)=\int_{0}^{+\infty} x^{r} f(x) d x \\
& =\sum_{i=0}^{\infty} \sum_{j=0}^{\infty} w_{i, j} \int_{0}^{+\infty} x^{r} h_{\beta(\alpha+i+j)}(x) d x \\
& =\sum_{i=0}^{\infty} \sum_{j=0}^{\infty} w_{i, j} \frac{(k+1) r^{s+k+1}}{r+k+1}
\end{aligned}
$$

The skewness and kurtosis measures can be calculated from the ordinary moments using well-known relationships. The $n$th central moment of $X$, say $M_{n}$ is

$$
M_{n}=E(X-\mu)^{n}=\sum_{h=0}^{n}(-1)^{h}\left(\begin{array}{l}
n \\
h
\end{array}\right)\left(\mu_{1}^{\prime}\right)^{n} \mu_{n-h}^{\prime} .
$$

The cumulants $\left(\kappa_{n}\right)$ of $X$ follow recursively from

$$
\kappa_{n}=\mu_{n}^{\prime}-\sum_{r=0}^{n-1}\left(\begin{array}{l}
n-1 \\
r-1
\end{array}\right) \kappa_{r} \mu_{n-r}^{\prime}
$$

where,

$\kappa_{1}=\mu_{1}^{\prime}, \kappa_{2}=\mu_{2}^{\prime}-\mu^{\prime}{ }_{1}^{2}, \kappa_{3}=\mu^{\prime}{ }_{3}-3 \mu^{\prime}{ }_{2} \mu^{\prime}{ }_{1}+\mu^{\prime}{ }_{1}{ }^{3}$,

etc.

The main application of the first incomplete moment refers to Bonferroniand Lorenz curves which are very useful in economics, reliability, demography, insurance and medicine. The answers to many important questions in economics require more than just knowing the mean of the distribution, its shape as well. The $s$ th incomplete moments, say $\varphi_{s}(t)$, is given by the following:

$$
\begin{aligned}
\varphi_{s}(t) & =\int_{0}^{t} x^{s} f(x) d x \\
& =\sum_{i=0}^{t} \sum_{j=0}^{\infty} w_{i, j} \int_{0}^{t} x^{s} h_{\beta(\alpha+i+j)}(x) d x \\
& =\sum_{i=0}^{\infty} \sum_{j=0}^{\infty} w_{i, j} \frac{(k+1) t^{s+k+1}}{s+k+1}
\end{aligned}
$$

The first incomplete of the GOGaU family, $\varphi_{1}(t)$, can be obtained by setting $s=1$ in (6).

In Figure 3, one can see 3-D surfaces of mean, variance, skewness and kurtosis of the GOGaU distribution.

\subsection{Entropy}

Entropy is an index for measuring variation or uncertainty of a random variable. The measure of entropy, Rrnyi [15], is d

$$
I_{R}(\gamma)=\frac{1}{1-\gamma} \log \left(\int_{0}^{\infty} f^{\gamma}(x) d x\right),
$$

for $\gamma>0$ and $\gamma \neq 1$.

The Shannon entropy measure is also defined by $E\{-\log [f(x)]\}$ that is a special state of the Rényi entropy when $\gamma \uparrow 1$, as the following:

$$
\begin{aligned}
f(x)^{\gamma} & =\left[\frac{\beta x^{\alpha \beta-1} e^{\frac{-x^{\beta}}{1-x^{\beta}}}}{\Gamma(\alpha)\left[1-x^{\beta}\right]^{\alpha+1}}\right]^{\gamma} \\
& =\frac{\beta^{\gamma} x^{\gamma(\alpha \beta-1)} e^{\frac{-x^{\beta}}{1-x^{\beta}}}}{[\Gamma(\alpha)]^{\gamma}\left[1-x^{\beta}\right]^{\gamma(\alpha+1)}} \\
& =\frac{\beta^{\gamma}}{[\Gamma(\alpha)]^{\gamma}} \sum_{i=0}^{\infty} \frac{(-1)^{i}}{i !} \gamma^{i} \frac{x^{\gamma(\alpha \beta-1)+\beta i} e^{\frac{-x^{\beta}}{1-x^{\beta}}}}{\left[1-x^{\beta}\right]^{\gamma(\alpha+1)+i}}
\end{aligned}
$$



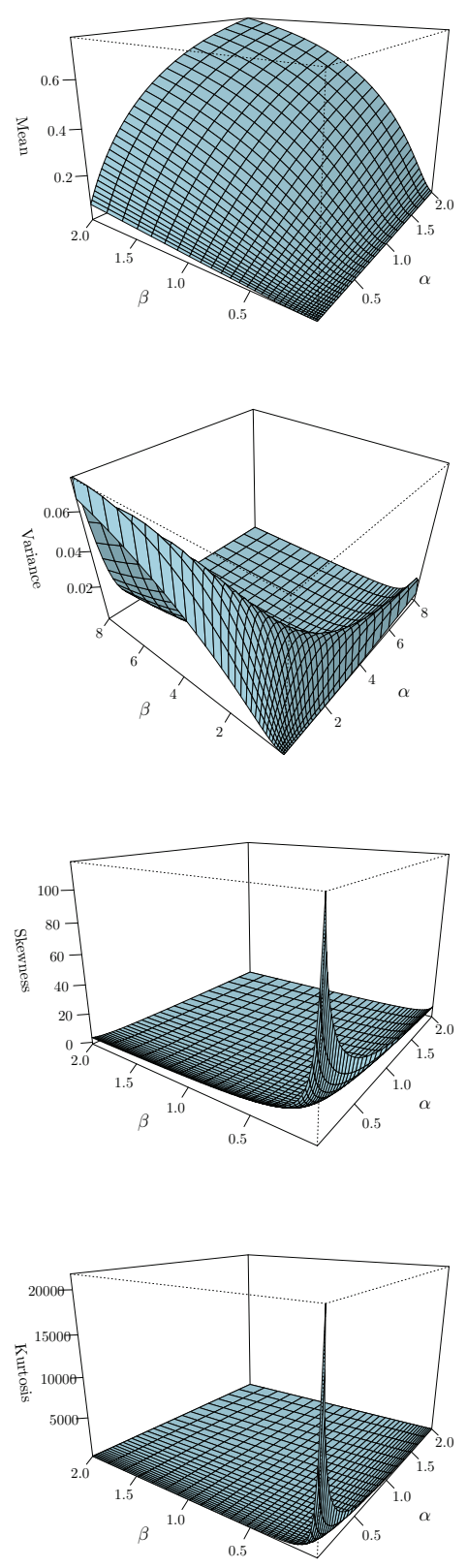

Figure 3: Surfaces of mean, variance, skewness and kurtosis for GOGaU.

$$
\begin{gathered}
=\frac{\beta^{\gamma}}{[\Gamma(\alpha)]^{\gamma}} \sum_{i=0}^{\infty} \sum_{j=0}^{\infty} \frac{(-1)^{i+j}}{i !} \\
\times\left(\begin{array}{c}
-\gamma(\alpha+1)-i \\
j
\end{array}\right) \gamma^{i} x^{\gamma(\alpha \beta-1)+\beta(i+j)} \\
\Rightarrow I_{R}(\gamma)=\frac{1}{1-\gamma} \log \left[\int_{-\infty}^{+\infty} f^{\gamma}(x) d x\right] \\
=\frac{\gamma}{1-\gamma} \log \left[\frac{\beta}{\Gamma(\alpha)}\right]
\end{gathered}
$$

$$
+\frac{1}{1-\gamma} \log \left[\sum_{i=0}^{\infty} \sum_{j=0}^{\infty} v_{i, j} I(\gamma, \alpha, \beta, i, j)\right]
$$

where

$$
\begin{gathered}
v_{i, j}=\frac{(-1)^{i+j} \gamma^{i}}{i !}\left(\begin{array}{c}
-\gamma(\alpha+1)-i \\
j
\end{array}\right) \\
I(\gamma, \alpha, \beta, i, j)=\int_{0}^{1} x^{\gamma(\alpha \beta-1)+\beta(i+j)} d x \\
=\frac{1}{\gamma(\alpha \beta-1)+\beta(i+j)+1} .
\end{gathered}
$$

In Figure 4 , one can see some 3-D surfaces of the entropy function of the GOGaU distribution.

\section{Estimation Methods}

\subsection{Maximum Likelihood Estimator}

The Maximum Likelihood Estimator (MLE) is one of the most common point estimators, and it is very applicable in confidence intervals and hypothesis testing. By MLE, various statistics are built for assessing the goodness-of-fit in a model, such as: the maximum log-likelihood $\left(\hat{\ell}_{\max }\right)$, Akaike Information Criterion (AIC), Bayesian Information Criterion (BIC), Anderson-Darling $\left(A^{*}\right)$ and Cramér-von Mises $\left(W^{*}\right)$, described by Chen and Balakrishnan [6]. The lower values of these statistics indicate that the model has better fitting. These statistics are used in section 5 .

For calculating the MLE, assuming that $x_{1}, x, \ldots, x_{n}$ are observations from pdf (2). In this case, by letting $\boldsymbol{\theta}=(\alpha, \beta)$ can be written as follows:

$$
\begin{aligned}
\ell_{n}(\boldsymbol{\theta})= & n \ln (\beta)+(\alpha \beta-1) \sum_{i=0}^{n} \ln \left(x_{i}\right)-\sum_{i=0}^{n} \frac{x_{i}{ }^{\beta}}{1-x_{i}{ }^{\beta}}-n \ln \\
& -(\alpha+1) \sum_{i=0}^{n} \ln \left(1-x_{i}{ }^{\beta}\right) .
\end{aligned}
$$

By numerically solving the following equations, the maximum likelihood estimators can be obtained.

$$
\left\{\begin{array}{l}
\frac{\partial \ell_{n}(\boldsymbol{\theta})}{\partial \alpha}=\beta \sum_{i=0}^{n} \ln \left(x_{i}\right)-n \frac{\Gamma^{\prime}(\alpha)}{\Gamma(\alpha)}+\sum_{i=0}^{n} \ln \left(1-x_{i}^{\beta}\right)=0 \\
\frac{\partial \ell_{n}(\boldsymbol{\theta})}{\partial \beta}=\frac{n}{\beta}+\alpha \sum_{i=0}^{n} \ln x_{i}-\sum_{i=0}^{n} \frac{x_{i}^{\beta} \ln x_{i}}{\left(1-x_{i}^{\beta}\right)^{2}} \\
+(\alpha+1) \sum_{i=0}^{n} \frac{x_{i}^{\beta} \ln x_{i}}{\left(1-x_{i}^{\beta}\right)}=0
\end{array}\right.
$$

\subsection{The Bayesian Estimators: Posterior Mean and Maximum-a-Posteriori}

As flexible statistical estimators, Bayesian estimators have significant applications in a wide variety of sta- 
tistical studies. They provide good estimation for parameters by combining past experience with current observations. In the following and Section 4, Posterior Mean (PM) and Maximum-a-Posteriori (MAP) estimator are examined to the estimation of distribution parameters. In Bayesian analysis after specifying the prior distribution of parameter, posterior distribution can be obtained as follows:

$$
\begin{aligned}
& \theta \sim \pi(\theta) \\
& f(\boldsymbol{x} \mid \theta)=\prod_{i=1}^{n} f\left(x_{i} \mid \theta\right) \\
& \pi(\theta \mid \boldsymbol{x})=c \pi(\theta) f(\boldsymbol{x} \mid \theta) ; c=\left(\int_{\Theta} \pi(\theta) f(\boldsymbol{x} \mid \theta) d \theta\right)^{-1}
\end{aligned}
$$

Now if one consider mean squared error loss function, Posterior Mean (PM) will be the bayes estimator. Also, the mode of posterior, Maximum-a-Posteriori (MAP), is other estimator that is equivalent to MLE in bayesian statistics; It means:

$$
\begin{gathered}
\mathrm{PM}=\mathrm{E}(\theta \mid \boldsymbol{x}) \\
\mathrm{MAP}=\underset{\theta}{\arg \max } \pi(\theta \mid \boldsymbol{x})
\end{gathered}
$$

\subsection{The other estimation methods}

There are several approaches to estimate the parameters of distributions and each has its own characteristic features and benefits. In this subsection five of those methods are briefly introduced and numerically investigated in the simulation study section (5). A useful summary of these methods can be seen in Dey et al. [11]. Here $\left\{t_{i} ; i=1,2, \ldots, n\right\}$ is the associated order statistics and $F$ is the distribution function of GOGaU.

\section{Least squares and weighted least squares estimators}

The Least Squares (LSE) and weighted Least Squares Estimators (WLSE) are introduced by Swain et al. [16]. The LSEs and WLSEs are obtained by minimizing the following functions:

$$
\begin{aligned}
S_{\mathrm{LSE}}(\alpha, \beta)=\sum_{i=1}^{n} & \left(F\left(t_{i} ; \alpha, \beta\right)-\frac{i}{n+1}\right)^{2} \\
S_{\mathrm{WLSE}}(\alpha, \beta)= & \sum_{i=1}^{n} \frac{(n+1)^{2}(n+2)}{i(n-i+1)} \\
& \times\left(F\left(t_{i} ; \alpha, \beta\right)-\frac{i}{n+1}\right)^{2}
\end{aligned}
$$

\section{Cramér-von-Mises estimator}

Cramér-von-Mises Estimator (CME) is introduced by Choi and Bulgren [7]. The CMEs is obtained by minimizing the following function:

$$
S_{\mathrm{CME}}(\alpha, \beta)=\frac{1}{12 n}+\sum_{i=1}^{n}\left(F\left(t_{i} ; \alpha, \beta\right)-\frac{2 i-1}{2 n}\right)^{2}
$$

\section{Anderson-Darling and right-tailed Anderson-Darling}

The Anderson-Darling (ADE) and Right-Tailed Anderson-Darling Estimators (RTADE) are introduced by Anderson and Darling [3]. The ADEs and RTADEs are obtained by minimizing the following functions:

$$
\begin{aligned}
S_{\mathrm{ADE}}(\alpha, \beta)= & -n-\frac{1}{n} \sum_{i=1}^{n}(2 i-1)\left\{\log F\left(t_{i} ; \alpha, \beta\right)\right. \\
& \left.+\log \bar{F}\left(t_{n+1-i} ; \alpha, \beta\right)\right\} \\
S_{\mathrm{RTADE}}(\alpha, \beta)= & \frac{n}{2}-2 \sum_{i=1}^{n} F\left(t_{i} ; \alpha, \beta\right) \\
& -\frac{1}{n} \sum_{i=1}^{n}(2 i-1) \log \bar{F}\left(t_{n+1-i} ; \alpha, \beta\right)
\end{aligned}
$$

where $\bar{F}(\cdot)=1-F(\cdot)$.

\section{Simulation study}

In this Section MLE, PM and MAP estimators are examined using pdf (2) simulation. In order to do that, two pdf of Figure 5 were selected in which two deferent cases of GOGaU distribution are visible: $(\alpha, \beta)=(1.1,0.45)$ skewed right and $(\alpha, \beta)=$ $(0.5,3.5)$ skewed left.

\subsection{MLE, Bayes and MAP}

To verify the validity of the estimators, Bias and Mean Square Error (MSE) of the Estimate have been used. For example, as described in Subsection 2.1, for $(\alpha, \beta)=(0.5,3.5)$, samples of $n=20,40,70,100,140,200,270,350,450,600$ of $\operatorname{GOGaU}(0.5,3.5)$ are generated for $N=5000$ times. For PM and MAP, the noninformative $\alpha, \beta \sim$ $\Gamma(0.01,0.01)$ is used. This prior has a large standard deviation and there is no information on the positive parameters used. According to Subsection 3.2 and using these priors, MAP and MLE produce similar results approximately. To obtain the posterior distribution we have the following relationships:

$\pi(\alpha, \beta)=\frac{0.01^{0.01}}{\Gamma(0.01)} \alpha^{-0.99} e^{-0.01 \alpha} \times \frac{0.01^{0.01}}{\Gamma(0.01)} \beta^{-0.99} e^{-0.01 \beta}$ 


$$
f\left(x_{i} \mid \alpha, \beta\right)=\frac{\beta x_{i}^{\alpha \beta-1} e^{\frac{-x_{i}^{\beta}}{1-x_{i}^{\beta}}}}{\Gamma(\alpha)\left(1-x_{i}^{\beta}\right)^{\alpha+1}}, i=1,2, \ldots n
$$

$\pi(\alpha, \beta \mid \boldsymbol{x}) \propto(\alpha \beta)^{-0.99} e^{-0.01(\alpha+\beta)} \prod_{i=1}^{n} \frac{\beta x_{i}^{\alpha \beta-1} e^{\frac{-x_{i}^{\beta}}{1-x_{i}^{\beta}}}}{\Gamma\left(1-x_{i}^{\beta}\right)^{\alpha+1}}$

$\pi(\alpha, \beta \mid \boldsymbol{x})$ is not a known distribution and the value of PM obtains using MCMC methods.

In this study, two different MCMC methods are applied and their gained results are compared. Perhaps, the most well-known method of MCMC would be Gibbs sampling that utilizes marginal densities to make simulation accurately. This method is technically applied in the two popular Bayesian software programs, WINDBAGS and JAGS, as well as many other relevant statistical packages. In this work, JAGS (Plummer [14]) is utilized. To define the new distribution in JAGS some techniques such as zero's trick that can be used, has remarkable Monte Carlo Error. The best way is to create new distribution module and launch it on JAGS (Wabersich and Vandekerckhove [17]); this module is made for GOGaU. rjags package needs to run JAGS and above mentioned module in $R$.

Furthermore, Hamiltonian Monte Carlo (HMC) is another method of MCMC simulation, applying Stan software (Carpenter et al. [5]), which is becoming more popular. In this method the gradient of the $\log$ posterior is utilized. Contrary to JAGS and WinBUGS, defining a new distribution in Stan software is more straightforward. rstan package needs to run Stan in $R$.

In order to obtain MAP estimator, Stan has a function using numerical methods (optimizing function in rstan), while in JAGS, some other method such as kernel curves must be used which cannot give as accurate result as Stan. JAGS and Stan model and some $R$ codes can be found in the Appendix A.

If $\boldsymbol{\theta}=(\alpha, \beta)$, for any simulation by $n$ volume and $i=1,2, \ldots, N$, the estimations are obtained as $\widehat{\boldsymbol{\theta}}_{i}=\left(\widehat{\alpha}_{i}, \widehat{\beta}_{i}\right)$. The standard deviation of estimators is obtained through the standard deviation of posterior distribution, because noninformative priors is used. The estimation of standard deviation is shown by $s_{\widehat{\boldsymbol{\theta}}_{i}}=\left(s_{\widehat{\alpha}_{i}}, s_{\widehat{\beta}_{i}}\right)$. In this case, the $\boldsymbol{\theta}$, Bias and MSE are calculated by the following formulas:

$$
\begin{gathered}
\widehat{\boldsymbol{\theta}}(n)=\frac{1}{N} \sum_{i=1}^{N} \widehat{\boldsymbol{\theta}}_{i} \\
\operatorname{Bias}_{\widehat{\boldsymbol{\theta}}}(n)=\frac{1}{N} \sum_{i=1}^{N}\left(\widehat{\boldsymbol{\theta}}_{i}-\boldsymbol{\theta}_{i}\right)
\end{gathered}
$$

$$
M S E_{\widehat{\boldsymbol{\theta}}}(n)=\frac{1}{N} \sum_{i=1}^{N}\left(\widehat{\boldsymbol{\theta}}_{i}-\boldsymbol{\theta}_{i}\right)^{2}
$$

Figures 6 represent the Biases and MSEs plots for $(\alpha, \beta)=(0.5,3.5)$. As expected, the biases and MSE of estimated parameters converge to zero while $n$ is growing. MLE and MAP is calculated in Stan estimation (MLE-Stan and MAP-Stan) are similar and PMStan and PM-JAGS estimation are almost equal. As we have already mentioned, the results of MAP-JAGS estimation are roughly different from MLE-Stan and MAP-Stan estimation. The plots of parameters vector $(\alpha, \beta)=(1.1,0.45)$ have the same position as one can see in Figures 10 in Appendix B.

\subsection{Simulation of other estimation methods}

In order to explore the introduced estimators in section 3.3, we consider the two models that have been used in the subsection 4.1, and we investigate Bias and MSE of those estimators for different samples. For instance according to what has been mentioned in the subsection 4.1, for $(\alpha, \beta)=(0.5,3.5)$ we have simulated $N=10000$ times with sample size of the $n=20,40,70,100,140,200,270,350,450,600$, then the Bias and MSE formulas that are mentioned in the subsection 4.1 are calculated for them. To obtain the value of the estimators, we have used the optim function and L-BFGS-B method in $R$.

The result of the simulations of this subsection is shown in Figure 77. As it is clear from the Bias and MSE plots for two parameters with the increase in the volume of the sample all methods will approach to zero; and this verifies the validity of the these estimation methods and numerical calculations for the distribution parameters GOGaU. The results of $(\alpha, \beta)=$ $(1.1,0.45)$ model in Figure 11 in Appendix $B$ have similar output.

The values of the confidence intervals along with coverage probabilities (CP) of the parameters based on different methods of estimation are obtained in Tables 1 and 2. Given that we used the Hessians matrix method to obtain the confidence interval, Some lower limits of confidence intervals that are negative can be considered zero.

\section{Application}

In this section, we present two applications by fitting the GOGaU and some famous models to a real data sets is assessed. The Akaike information criterion (AIC), Bayesian information criterion (BIC), Anderson-Darling $\left(A^{*}\right)$ and Cramér-von Mises $\left(W^{*}\right)$, Kolmogorov-Smirnov (K.S) and the P-Value of K.S test, are utilized for comparison of the models. The distributions: Beta (B), Kumaraswamy (Kw) (Kumaraswamy [13]), Kumaraswamy Beta (KwB) 
Table 1: Confidence intervals and coverage probability for selected parameters $(\alpha, \beta)=(0.5,3.5)$.

\begin{tabular}{lclclclc} 
Method & $(\alpha, \beta)$ & $n=100$ & $\mathrm{CP}$ & $n=200$ & $\mathrm{CP}$ & $n=300$ & $\mathrm{CP}$ \\
\hline MLE & $\alpha$ & $(0.222,0.863)$ & 0.958 & $(0.299,0.742)$ & 0.956 & $(0.331,0.687)$ & 0.945 \\
& $\beta$ & $(1.587,5.420)$ & 0.904 & $(2.165,4.871)$ & 0.935 & $(2.428,4.647)$ & 0.952 \\
\hline PM(Stan) & $\alpha$ & $(0.244,0.864)$ & 0.934 & $(0.308,0.741)$ & 0.935 & $(0.338,0.687)$ & 0.941 \\
& $\beta$ & $(2.316,8.232)$ & 0.935 & $(2.570,5.759)$ & 0.930 & $(2.710,5.137)$ & 0.946 \\
\hline PM(JAGS) & $\alpha$ & $(0.254,0.867)$ & 0.938 & $(0.317,0.743)$ & 0.920 & $(0.345,0.694)$ & 0.937 \\
& $\beta$ & $(2.312,7.771)$ & 0.936 & $(2.558,5.624)$ & 0.924 & $(2.692,5.073)$ & 0.933 \\
\hline MAP(Stan) & $\alpha$ & $(-0.065,1.149)$ & 0.990 & $(0.089,0.951)$ & 0.998 & $(0.162,0.867)$ & 0.996 \\
& $\beta$ & $(2.997,4.069)$ & 0.412 & $(3.13,3.89)$ & 0.431 & $(3.193,3.814)$ & 0.421 \\
\hline MAP(JAGS) & $\alpha$ & $(0.412,0.536)$ & 0.753 & $(0.458,0.519)$ & 0.830 & $(0.464,0.523)$ & 0.833 \\
& $\beta$ & $(3.065,3.850)$ & 0.860 & $(3.277,3.650)$ & 0.935 & $(3.289,3.643)$ & 0.866 \\
\hline LSE & $\alpha$ & $(-1.440,2.478)$ & 1 & $(-0.860,1.871)$ & 1 & $(-0.606,1.609)$ & 1 \\
& $\beta$ & $(-11.660,19.614)$ & 1 & $(-5.885,13.345)$ & 1 & $(-3.910,11.247)$ & 1 \\
\hline WLSE & $\alpha$ & $(0.463,0.595)$ & 0.273 & $(0.479,0.543)$ & 0.197 & $(0.486,0.528)$ & 0.155 \\
& $\beta$ & $(3.286,4.167)$ & 0.290 & $(3.408,3.823)$ & 0.203 & $(3.448,3.719)$ & 0.169 \\
\hline CME & $\alpha$ & $(-1.473,2.583)$ & 1 & $(-0.866,1.912)$ & 1 & $(-0.608,1.634)$ & 1 \\
& $\beta$ & $(-10.196,17.620)$ & 1 & $(-5.516,12.737)$ & 1 & $(-3.735,10.917)$ & 1 \\
\hline ADE & $\alpha$ & $(-0.118,1.170)$ & 0.999 & $(0.061,0.958)$ & 0.999 & $(0.141,0.870)$ & 0.999 \\
& $\beta$ & $(-0.700,8.128)$ & 0.999 & $(0.674,6.568)$ & 0.994 & $(1.227,5.950)$ & 0.994 \\
RTADE & $\alpha$ & $(-0.118,1.170)$ & 0.998 & $(0.061,0.958)$ & 0.999 & $(0.141,0.870)$ & 0.999 \\
& $\beta$ & $(-0.700,8.128)$ & 0.999 & $(0.674,6.568)$ & 1 & $(1.227,5.950)$ & 0.999
\end{tabular}

Table 2: Confidence intervals and coverage probability for selected parameters $(\alpha, \beta)=(1.1,0.45)$.

\begin{tabular}{lclclccc} 
Method & $(\alpha, \beta)$ & $n=100$ & $\mathrm{CP}$ & $n=200$ & $\mathrm{CP}$ & $n=300$ & $\mathrm{CP}$ \\
\hline MLE & $\alpha$ & $(0.615,1.712)$ & 0.943 & $(0.750,1.514)$ & 0.944 & $(0.807,1.424)$ & 0.953 \\
& $\beta$ & $(0.253,0.649)$ & 0.919 & $(0.311,0.589)$ & 0.932 & $(0.338,0.564)$ & 0.953 \\
\hline PM(Stan) & $\alpha$ & $(0.645,1.713)$ & 0.937 & $(0.759,1.506)$ & 0.951 & $(0.816,1.422)$ & 0.950 \\
& $\beta$ & $(0.315,0.794)$ & 0.948 & $(0.346,0.650)$ & 0.945 & $(0.362,0.601)$ & 0.942 \\
\hline PM(JAGS) & $\alpha$ & $(0.656,1.712)$ & 0.936 & $(0.766,1.504)$ & 0.935 & $(0.817,1.416)$ & 0.919 \\
& $\beta$ & $(0.293,0.659)$ & 0.939 & $(0.347,0.647)$ & $(0.933)$ & $(0.364,0.602)$ & 0.930 \\
\hline MAP(Stan) & $\alpha$ & $(0.679,1.641)$ & 0.908 & $(0.794,1.475)$ & 0.914 & $(0.848,1.404)$ & 0.922 \\
& $\beta$ & $(0.017,0.882)$ & 1 & $(0.142,0.754)$ & 1 & $(0.198,0.697)$ & 0.999 \\
\hline MAP(JAGS) & $\alpha$ & $(0.982,1.167)$ & 0.903 & $(1.023,1.140)$ & 0.900 & $(1.041,1.141)$ & 0.897 \\
& $\beta$ & $(0.432,0.445)$ & 0.901 & $(0.426,0.467)$ & 0.867 & $(0.428,0.465)$ & 0.869 \\
\hline LSE & $\alpha$ & $(-1.917,4.135)$ & 1 & $(-1.013,3.205)$ & 1 & $(-0.625,2.817)$ & 1 \\
& $\beta$ & $(-0.784,1.751)$ & 1 & $(-0.378,1.317)$ & 1 & $(-0.212,1.139)$ & 1 \\
\hline WLSE & $\alpha$ & $(1.020,1.229)$ & 0.255 & $(1.058,1.160)$ & 0.423 & $(1.071,1.139)$ & 0.168 \\
& $\beta$ & $(0.429,0.511)$ & 0.245 & $(0.441,0.480)$ & 0.513 & $(0.444,0.470)$ & 0.159 \\
\hline CME & $\alpha$ & $(-1.950,4.281)$ & 1 & $(-1.016,3.263)$ & 1 & $(-0.623,2.851)$ & 1 \\
& $\beta$ & $(-0.722,1.644)$ & 1 & $(-0.360,1.279)$ & 1 & $(-0.204,1.17)$ & 1 \\
\hline ADE & $\alpha$ & $(0.097,2.155)$ & 1 & $(0.386,1.827)$ & 0.999 & $(0.516,1.691)$ & 0.998 \\
& $\beta$ & $(0.063,0.873)$ & 0.990 & $(0.183,0.740)$ & 0.994 & $(0.234,0.681)$ & 0.996 \\
\hline RTADE & $\alpha$ & $(0.097,2.155)$ & 1 & $(0.386,1.827)$ & 1 & $(0.516,1.691)$ & 1 \\
& $\beta$ & $(0.063,0.873)$ & 0.999 & $(0.674,6.568)$ & 1 & $(0.234,0.681)$ & 1
\end{tabular}

(Cordeiro and Castro [9]), Beta (BB) (Zografos and Balakrishnan [19]), McDonald Beta (McB) (Alexander et al. [1]]), Exponentiated Gompertz Generated Beta (EGGB) (Cordeiro et al.[8]), beta power (BP) (Cordeiro et al.[10]), Logistic-normal (LogitN) (Atchison and Shen [4]), are selected for comparison. The parameters of models are estimated by the MLE method using optimizing function in rstan package in $R$.

\subsection{The total milk production data set}

This subsection is related to study of the total milk production in 107 SINDI race cows on the first birth after to calve which presented by EGGB that include 107 observations. The data set is:

$0.4365,0.4260,0.5140,0.6907,0.7471,0.2605$, $0.6196,0.8781,0.4990,0.6058,0.6891,0.5770$, $0.5394,0.1479,0.2356,0.6012,0.1525,0.5483$, $0.6927,0.7261,0.3323,0.0671,0.2361,0.4800$, $0.5707,0.7131,0.5853,0.6768,0.5350,0.4151$, $0.6789,0.4576,0.3259,0.2303,0.7687,0.4371$, $0.3383,0.6114,0.3480,0.4564,0.7804,0.3406$,
$0.4823,0.5912,0.5744,0.5481,0.1131,0.7290$, $0.0168,0.5529,0.4530,0.3891,0.4752,0.3134$, $0.3175,0.1167,0.6750,0.5113,0.5447,0.4143$, $0.5627,0.5150,0.0776,0.3945,0.4553,0.4470$, $0.5285,0.5232,0.6465,0.0650,0.8492,0.8147$, $0.3627,0.3906,0.4438,0.4612,0.3188,0.2160$, $0.6707,0.6220,0.5629,0.4675,0.6844,0.3413$, $0.4332,0.0854,0.3821,0.4694,0.3635,0.4111$, $0.5349,0.3751,0.1546,0.4517,0.2681,0.4049$, $0.5553,0.5878,0.4741,0.3598,0.7629,0.5941$, $0.6174,0.6860,0.0609,0.6488,0.2747$

The Tables 3 and 4 display a summary of the fitted information criteria and MLEs for this data with different models, respectively. Models have been sorted from the lowest to the highest value of AIC. As it is visible, the GOGaU is selected as the best model with all the criteria. Note that P-Value for GOGaU is also more than all other distributions. The histogram of data set and the plots of fitted pdf are displayed in Figure 8.

Table 3: Information criteria for the total milk production data set.

\begin{tabular}{lllllll} 
Model & AIC & BIC & $W^{*}$ & $A^{*}$ & K.S & P-Value \\
\hline GOGaU & -50.38 & -45.04 & 0.094 & 0.572 & 0.068 & 0.70 \\
\hline KwB & -48.80 & -38.11 & 0.067 & 0.423 & 0.078 & 0.541 \\
\hline BB & -47.97 & -37.28 & 0.081 & 0.513 & 0.071 & 0.657 \\
\hline BP & -47.54 & -36.85 & 0.083 & 0.531 & 0.073 & 0.614 \\
\hline EGGB & -46.91 & -33.55 & NaN & NaN & 1 & 0.000 \\
\hline Kw & -46.79 & -41.44 & 0.156 & 1.009 & 0.076 & 0.563 \\
\hline McB & -46.00 & -32.64 & 0.065 & 0.422 & 0.372 & 0.000 \\
\hline Beta & -43.55 & -38.21 & 0.208 & 1.326 & 0.091 & 0.338 \\
\hline LN & -37.03 & -31.68 & 0.316 & 1.924 & 0.108 & 0.165
\end{tabular}

Table 4: MLEs for the total milk production data set.

\begin{tabular}{ll} 
Model & Parameters \\
\hline GOGaU & $(\widehat{\alpha}, \widehat{\beta})=(1.61,0.80)$ \\
& $\left(s_{\widehat{\alpha}}, s_{\widehat{\beta}}\right)=(0.211,0.193)$ \\
\hline KwB & $(\widehat{c}, \widehat{b}, \widehat{\alpha}, \widehat{\beta})=(0.09,0.19,18.35,24.78)$ \\
& $\left(s_{\widehat{c}}, s_{\widehat{b}}, s_{\widehat{\alpha}}, s_{\widehat{\beta}}\right)=(0.469, N a N, 0.356, N a N)$ \\
\hline BB & $(\widehat{a} 1, \widehat{b 1}, \widehat{a 2}, \hat{b 2})=(0.44,33648.89,3.79,0.0002)$ \\
& $\left(s_{\widehat{a 1}}, s_{\widehat{b 1}}, s_{\widehat{a 2}}, s_{\widehat{b 2}}\right)=(0.449,26.699,0.372,53.454)$ \\
\hline BP & $(\widehat{a}, b, \widehat{\alpha}, \beta)=(0.26,59295.17,6.86,0.27)$ \\
& $\left(s_{\widehat{a}}, s_{\widehat{b}}, s_{\widehat{\alpha}}, s_{\widehat{\beta}}\right)=(0.363,130.138,0.284,24.970)$ \\
\hline EGGB & $(\widehat{a}, \hat{b}, \widehat{c}, \widehat{\alpha}, \widehat{\beta})=(0.09,0.18,22.00,0.0001,3.16)$ \\
& $\left(s_{\widehat{a}}, s_{\widehat{b}}, s_{\widehat{c}}, s_{\widehat{\alpha}}, s_{\widehat{\beta}}\right)=(3.510,2.460,1.581,40.381,2.075)$ \\
\hline Kw & $(\widehat{\alpha}, \widehat{\beta})=(2.41,2.83)$ \\
& $\left(s_{\widehat{\alpha}}, s_{\widehat{\beta}}\right)=(0.101,0.169)$ \\
\hline McB & $(\widehat{a}, \widehat{b}, \widehat{c}, \widehat{\alpha}, \widehat{\beta})=(0.52,2192324.30,1.44,2.15,0.0001)$ \\
& $\left(s_{\widehat{a}}, s_{\widehat{b}}, s_{\widehat{c}}, s_{\widehat{\alpha}}, s_{\widehat{\beta}}\right)=(0.536,4.223,0.375,0.761, N a N)$ \\
\hline Beta & $(\widehat{\alpha}, \widehat{\beta})=(2.19,3.44)$ \\
& $\left(s_{\widehat{\alpha}}, s_{\widehat{\beta}}\right)=(0.130,0.132)$ \\
\hline LN & $(\widehat{\mu}, \widehat{\sigma})=(-0.19,0.99)$ \\
& $\left(s_{\widehat{\mu}}, s_{\widehat{\sigma}}\right)=(0.096,0.068)$ \\
&
\end{tabular}




\subsection{The time to failure $\left(10^{3} \mathbf{h}\right)$ of turbocharger data set}

This subsection is related to study of the time to failure $\left(10^{3} \mathrm{~h}\right)$ of turbocharger of one type of engine which presented by $\mathrm{Xu}$ et al. [18] that include 40 observations. The data set divided to 10 are: 0.16 $, 0.35,0.48,0.54,0.60,0.65,0.70,0.73,0.77,0.80$, $0.84,0.20,0.39,0.50,0.56,0.61,0.65,0.71,0.73$, $0.78,0.81,0.84,0.26,0.45,0.51,0.58,0.63,0.67$, $0.73,0.77,0.79,0.83,0.85,0.30,0.46,0.53,0.60$, $0.87,0.88,0.90$. Similar to the previous application example, we have Tables 5 and 6 . As it is clear, the GOGaU is selected as the best model with more criteria. The histogram of the Time to failure $\left(10^{3} \mathrm{~h}\right)$ of turbocharger data and the plots of fitted pdf are displayed in Figure 9 .

Table 5: Information criteria for the time to failure $\left(10^{3} \mathrm{~h}\right)$ of turbocharger data set.

\begin{tabular}{lllllll} 
Model & AIC & BIC & $W^{*}$ & $A^{*}$ & K.S & P-Value \\
\hline GOGaU & -24.06 & -20.68 & 0.015 & 0.104 & 0.057 & 0.999 \\
\hline LN & -21.80 & -18.42 & 0.030 & 0.243 & 0.075 & 0.977 \\
\hline BB & -21.16 & -14.40 & 0.011 & 0.827 & 0.048 & 1 \\
\hline BP & -21.14 & -14.38 & 0.012 & 0.090 & 0.055 & 1 \\
\hline Kw & -20.69 & -17.31 & 0.036 & 0.288 & 0.087 & 0.920 \\
\hline Beta & -20.01 & -16.63 & NaN & NaN & 1.466 & 0.000 \\
\hline KwB & -19.29 & -12.53 & 0.017 & 0.126 & 0.068 & 0.993 \\
\hline EGGB & -19.15 & -10.71 & NaN & NaN & 1 & 0.000 \\
\hline McB & -18.92 & -10.47 & 0.144 & 0.851 & 0.735 & 0.000
\end{tabular}

Table 6: MLEs for the time to failure $\left(10^{3} \mathrm{~h}\right)$ of turbocharger data set.

\begin{tabular}{|c|c|}
\hline Model & Parameters \\
\hline GOGaU & $\begin{array}{l}(\widehat{\alpha}, \widehat{\beta})=(1.07,2.05) \\
\left(s_{\widehat{\alpha}}, s_{\widehat{\beta}}\right)=(0.419,0.723)\end{array}$ \\
\hline LN & $\begin{array}{l}(\widehat{\mu}, \widehat{\sigma})=(0.59,0.92) \\
\left(s_{\widehat{\mu}}, s_{\widehat{\sigma}}\right)=(0.145,0.103)\end{array}$ \\
\hline BB & $\begin{array}{l}(\overrightarrow{a 1}, \overrightarrow{b 1}, \widehat{a 2}, \widehat{2})=(0.03,71.71,98.37,4.42) \\
\left(s_{\widehat{a 1}}, s_{\widehat{b 1}}, s_{\widehat{a 2}}, s_{\widehat{b 2}}\right)=(0.060,396.959,241.232,15.868)\end{array}$ \\
\hline BP & $\begin{array}{l}(\widehat{a}, \vec{b}, \widehat{\alpha}, \beta)=(0.03,8.04,74.96,1.08) \\
\left(s_{\widehat{a}}, s_{\widehat{b}}, s_{\widehat{\alpha}}, s_{\widehat{\beta}}\right)=(0.087, N a N, 202.013,0.031)\end{array}$ \\
\hline $\mathrm{Kw}$ & $\begin{array}{l}(\widehat{\alpha}, \widehat{\beta})=(3.16,2.49) \\
\left(s_{\widehat{\alpha}}, s_{\widehat{\beta}}\right)=(0.549,0.636)\end{array}$ \\
\hline Beta & $\begin{array}{l}(\widehat{\alpha}, \widehat{\beta})=(3.57,2.19) \\
\left(s_{\widehat{\alpha}}, s_{\widehat{\beta}}\right)=(0.787,0.462)\end{array}$ \\
\hline KwB & $\begin{array}{l}(\widehat{c}, \widehat{b}, \widehat{\alpha}, \widehat{\beta})=(21.12,60.90,0.09,0.30) \\
\left(s_{\widehat{c}}, s_{\widehat{b}}, s_{\widehat{\alpha}}, s_{\widehat{\beta}}\right)=(36.915,142.856,0.159,0.200)\end{array}$ \\
\hline EGGB & $\begin{array}{l}(\widehat{a}, \widehat{b}, \widehat{c}, \widehat{\alpha}, \widehat{\beta})=(1.41,2.62,0.16,15.80,2.09) \\
\left(s_{\widehat{a}}, s_{\widehat{b}}, s_{\widehat{c}}, s_{\widehat{\alpha}}, s_{\widehat{\beta}}\right)=(0.555,1.267,0.034,1.104,0.422)\end{array}$ \\
\hline $\mathrm{McB}$ & $\begin{array}{l}(\widehat{a}, \widehat{b}, \widehat{c}, \widehat{\alpha}, \widehat{\beta})=(0.04,26.13,3.57,17.68,1.86) \\
\left(s_{\widehat{a}}, s_{\widehat{b}}, s_{\widehat{c}}, s_{\widehat{\alpha}}, s_{\widehat{\beta}}\right)=(0.071,61.136,16.069,86.688,3.932\end{array}$ \\
\hline
\end{tabular}

\section{Conclusions}

In this paper, we introduce a new two-parameter of Generalized Odd Gamma distribution on $(0,1)$ such as (GOGaU) family of distributions. Some mathematical and Various properties of the new class are provided.

Comparing the performances of the estimators such as: MLE, PM and MAP (Stan and JAGS), LSE, WLSE, CME, ADE and RTADE are proposed. We conclude from the Bias and MSE plots for two parameters of $\mathrm{GOGaU}$ with the increase in the sample size, all methods will approach to zero and this verifies the validity of the these estimation methods. The GOGaU module is build for using in JAGS models.

The (GOGaU) distribution is applied to fit a real data sets. These applications show that the model has the ability to fit left-skewed and heavy-tailed data due to its flexibility. The results of tables and figures illustrate the importance of the new distribution to analyze of real data with respect to another nested well-known models.

\section{acknowledgements}

We would like to show our gratitude to the Prof. Vandekerckhove (University of California, Irvine) and Dr. Dominik Wabersich for updating and sharing their article details. It was not possible to build GOGaU JAGS module without their collaboration.

Also, the support of Research Committee of Persian Gulf University is greatly acknowledged.

\section{References:}

[1] Alexander, C., Cordeiro, G.-M., Ortega, E.-M.M., Sarabia, G. M.: Generalized beta-generated distributions. Computational Statistics \& Data Analysis. 56(6), 1880-1897 (2012)

[2] Alzaatreh, A., Lee, C., Famoye, F.: A new method for generating families of continuous distributions. Metron. 71(1), 63-79 (2013)

[3] Anderson, T.-W., Darling, D.-A.: Asymptotic theory of certain" goodness of fit" criteria based on stochastic processes. The annals of mathematical statistics. 193-212 (1952)

[4] Atchison, J., Shen, S.-M.: Logistic-normal distributions: Some properties and uses. Biometrika. 67(2) 261-272 (1980)

[5] Carpenter, B., Gelman, A., Hoffman, M., Lee, D., Goodrich, B., Betancourt, M., Brubaker, M., Guo, J., Li, P., Riddell, A.: Stan: A probabilistic programming language. Journal of Statistical Software. 20(2), 1-37 (2016) 
[6] Chen, G., Balakrishnan, N.: A general purpose approximate goodness-of-fit test. Journal of Quality Technology. 27(2)154-161 (1995)

[7] Choi, K., Bulgren, W.-G.: An estimation procedure for mixtures of distributions. Journal of the Royal Statistical Society. Series B (Methodological). 444-460 (1968)

[8] Cordeiro, G.-M., Alizadeh, M., Nascimento, A.D.-C., Rasekhi, M.: The exponentiated gompertz generated family of distributions: Properties and applications. Chilean Journal of Statistics (ChJS). 7(2) (2016)

[9] Cordeiro, G.-M., de Castro, M.: A new family of generalized distributions. Journal of statistical computation and simulation. 81(7), 883-898 (2011)

[10] Cordeiro, G.-M., dos Santos, R., Brito, E.-D.: The beta power distribution. Brazilian journal of probability and statistics. 26(1), 88-112 (2012)

[11] Dey, S., Mazucheli, J., Nadarajah, S.: Kumaraswamy distribution: different methods of estimation. Computational and Applied Mathematics. 1-18 (2017)

[12] Hosseini, B., Afshari, M., Alizadeh, M.: The generalized odd gamma-g family of distributions: Properties and applications. AJS. 47, 69-89 (2018)

[13] Kumaraswamy, P.: A generalized probability density function for double-bounded random processes. Journal of Hydrology. 46(1-2) , 79-88 (1980)

Journal of the Royal Statistical Society: Series B (Statistical Methodology). 62(2) 271-292 (2000)

[14] Plummer. M.: Jags: just another gibbs sampler proceedings of the 3 rd international workshop on distributed statistical computing (dsc 2003). Vienna, Austria. (2005)

[15] Rrnyi, A.: On measures of entropy and information. In Fourth Berkeley symposium on mathematical statistics and probability. 1, 547561 (1961).

[16] Swain, J., Venkatraman, S., Wilson, J.-R.: Least-squares estimation of distribution functions in johnson's translation system. Journal of Statistical Computation and Simulation. 29(4), 271297 (1988)

[17] Wabersich, D., Vandekerckhove, J.: Extending jags: A tutorial on adding custom distributions to jags (with a diffusion model example). Behavior research methods. 46(1), 15-28 (2014)
[18] Xu, K., Xie, M., Tang, L.-C., Ho, S.-L.: Application of neural networks in forecasting engine systems reliability. Applied Soft Computing. 2, 255-268 (2003)

[19] Zografos, K., Balakrishnan, K.: On families of beta and generalized gamma-generated distributions and associated inference. Statistical Methodology. 6(4), 344-362 (2009)

\section{Appendices}

\section{A The $R$ codes of GOGaU}

The program is developed in $\mathrm{R}$ to obtain the value of density (dGOGaU), distribution (pGOGaU), hazard (hGOGaU), quantile (qGOGaU) function and random generation (rGOGaU) for the GOGaU distribution.

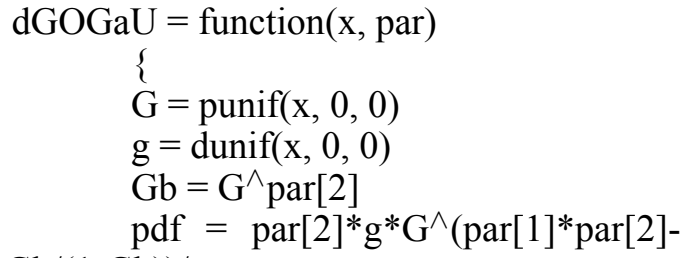

$$
\begin{aligned}
& 1) * \exp (-\mathrm{Gb} /(1-\mathrm{Gb})) / \\
& \left.\mathrm{Gb})^{\wedge}(\operatorname{par}[1]+1)\right) \\
& \text { pdf[! is.finite(pdf) }]=\text { NA } \\
& \text { pdf } \\
& \text { \} \# end of dGOGaU } \\
& \text { pGOGaU }=\text { function }(x, \text { par }) \\
& \{ \\
& \mathrm{G}=\operatorname{punif}(\mathrm{x}, 0,0) \\
& \mathrm{g}=\operatorname{dunif}(\mathrm{x}, 0,0) \\
& \mathrm{Gb}=\mathrm{G}^{\wedge} \operatorname{par}[2] \\
& \mathrm{cdf}=\operatorname{pgamma}(\mathrm{Gb} /(1-\mathrm{Gb}), \operatorname{par}[1], 1) \\
& \text { cdf[! is.finite(cdf)] }=\text { NA } \\
& \text { cdf } \\
& \text { \} \# end of pGOGaU } \\
& \mathrm{qGOGaU}=\text { function(p, par) } \\
& \{ \\
& \mathrm{a}=\text { qgamma }(\mathrm{p}, \operatorname{par}[1], 1) \\
& \mathrm{b}=(\mathrm{a} /(1+\mathrm{a}))^{\wedge}(1 / \operatorname{par}[2]) \\
& \text { qunif(b, } 0,1) \\
& \text { \} \# end of qGOGaU } \\
& \mathrm{hGOGaU}=\text { function }(\mathrm{x}, \mathrm{par}) \\
& \{ \\
& \mathrm{pdf}=\mathrm{dGOGaU}(\mathrm{x}=\mathrm{x}, \mathrm{par}=\mathrm{par}) \\
& \mathrm{cdf}=\mathrm{pGOGaU}(\mathrm{x}=\mathrm{x}, \mathrm{par}=\mathrm{par}) \\
& \mathrm{hrf}=\mathrm{pdf} /(1-\mathrm{cdf}) \\
& \text { hrf[! is.finite }(\mathrm{hrf})]=\mathrm{NA} \\
& \text { hrf } \\
& \text { \} \# end of hGOGaU }
\end{aligned}
$$




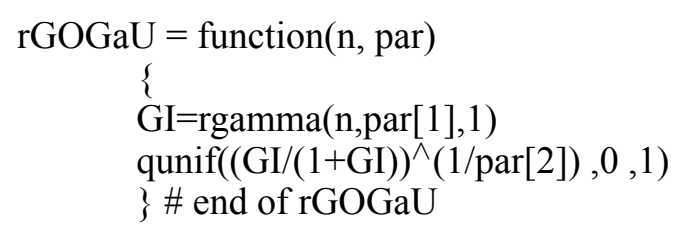

The program is developed in $\mathrm{R}$ of calculation for one-dimensional integral based on observations and the trapezoidal rule integration:

$$
\begin{gathered}
\text { intob }=\text { function }(x, y) \quad 0.5 * \operatorname{sum}\left(\operatorname{diff}(x)^{*}\right. \\
1 \text { 1 }]+y[1: \operatorname{length}(x)-
\end{gathered}
$$

The program is developed in $\mathrm{R}$ of calculation for the value of Rényi entropy:

$$
\begin{aligned}
& \text { REntropy }=\text { function (par, gamma) } \\
& \text { fgamma }=\text { function }(x) d G O G a U(x, \\
& \text { par }=\text { par })^{\wedge} \text { gamma } \\
& x=\operatorname{seq}(0,1,1 e=10000) \\
& \mathrm{y}=\operatorname{fgamma}(\mathrm{x}) \\
& \text { ent }=\log (\text { intob }(\mathrm{x}, \mathrm{y})) /(1 \text {-gamma }) \\
& \text { ent }=\text { ent[! is.finite }(\mathrm{ent})]=\mathrm{NA} \\
& \text { return(ent) } \\
& \text { \} \# end of REntropy }
\end{aligned}
$$

The program is developed in $\mathrm{R}$ of calculation for the value of moment, skewness and kurtosis:

$$
\begin{aligned}
&\left.\left.\left.\mathrm{m} 1)^{\wedge} 3^{*} \mathrm{y}\right)\right) / \operatorname{sqrt}(\mathrm{m} 2)^{\wedge} 3\right) \\
&\} \# \text { end of skew } \\
& \mathrm{kurt}= \text { function }(\text { par }) \\
&\{ \\
& \mathrm{x}=\mathrm{seq}(0,1, \mathrm{e}=10000) \\
& \mathrm{y}=\mathrm{dGOGaU}(\mathrm{x}=\mathrm{x}, \text { par }=\text { par }) \mathrm{m} 1=\operatorname{intob}(\mathrm{x}, \mathrm{x} * \mathrm{y}) \\
& \mathrm{m} 2=\operatorname{intob}\left(\mathrm{x},(\mathrm{x}-\mathrm{m} 1)^{\wedge} 2^{*} \mathrm{y}\right) \\
& \text { return(intob }(\mathrm{x},
\end{aligned}
$$$$
\text { moment }=\text { function }(\text { par, order })
$$$$
\{
$$$$
\mathrm{x}=\operatorname{seq}(\operatorname{par}[3], \operatorname{par}[4], \mathrm{le}=10000)
$$$$
\mathrm{y}=\mathrm{dGOGaU}(\mathrm{x}=\mathrm{x}, \mathrm{par}=\text { par })
$$$$
\text { return(intob(x, } \left.\left.\mathrm{x}^{\wedge} \text { order } * \mathrm{y}\right)\right)
$$$$
\text { \} \# end of moment }
$$$$
\text { skew }=\text { function(par) }
$$$$
\{
$$$$
\mathrm{x}=\operatorname{seq}(0,1,1 \mathrm{e}=10000)
$$$$
\mathrm{y}=\mathrm{dGOGaU}(\mathrm{x}=\mathrm{x}, \text { par }=\text { par })
$$$$
\mathrm{m} 1=\operatorname{intob}\left(\mathrm{x}, \mathrm{x}^{*} \mathrm{y}\right)
$$$$
\mathrm{m} 2=\operatorname{intob}\left(\mathrm{x},(\mathrm{x}-\mathrm{m} 1)^{\wedge} 2 * \mathrm{y}\right)
$$$$
\text { return(intob(x, }
$$

$$
\begin{array}{r}
\left.\left.\mathrm{m} 1)^{\wedge} 4^{*} \mathrm{y}\right) / \mathrm{sqrt}(\mathrm{m} 2)^{\wedge} 4\right) \\
\} \# \text { end of kurt }
\end{array}
$$

JAGS model and codes for GOGaU distribution. model

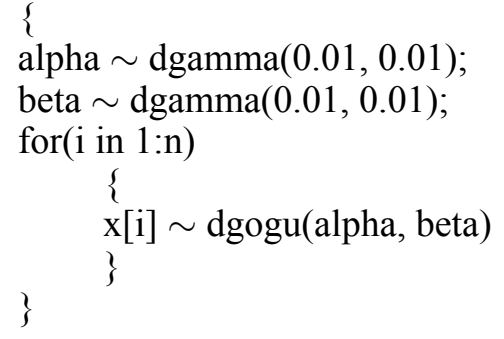

Stan model and codes for GOGaU distribution using optimization for simulations and application.

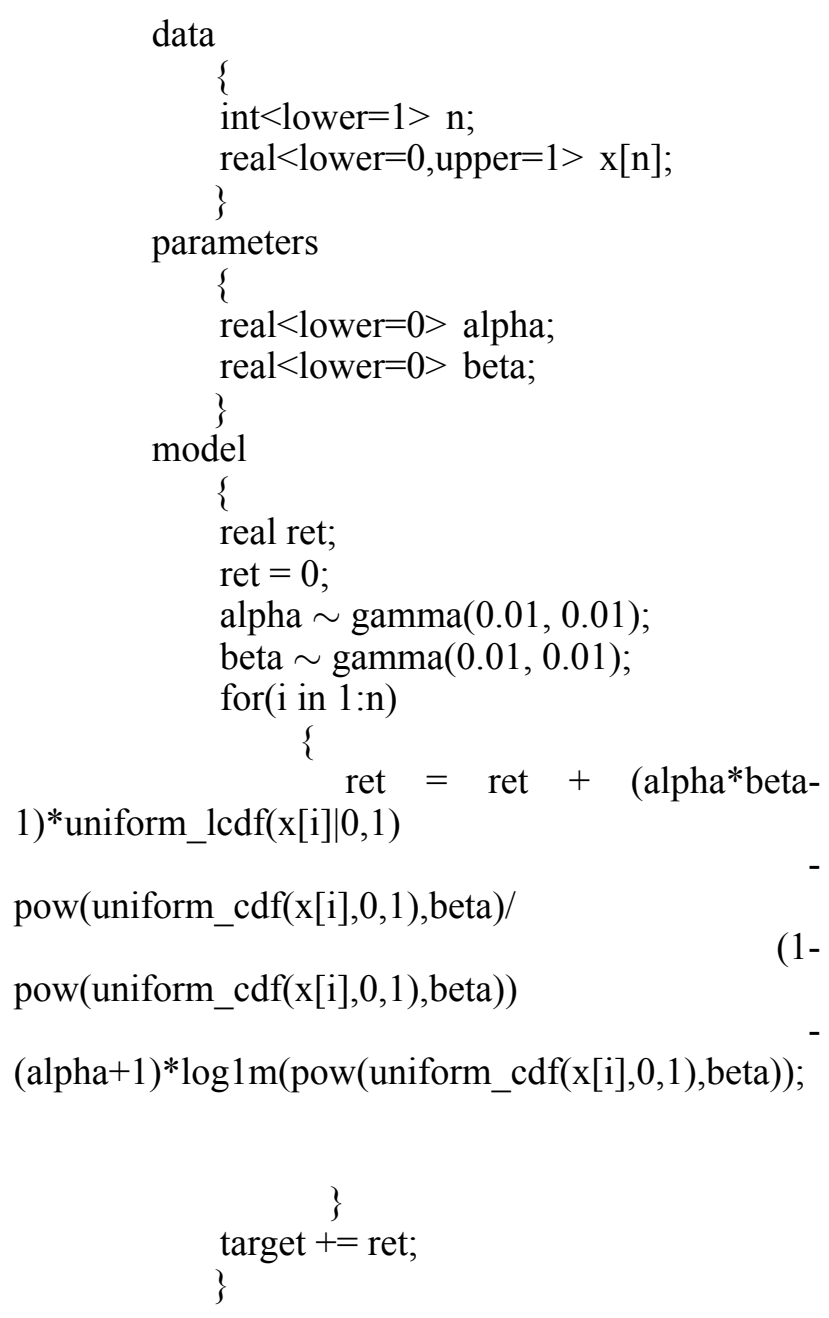

After saving the above code in "GOGaUModel.stan" file, one can use following rstan codes for simulation and optimization of posterior.

$$
\text { mystanGOGaU }
$$

stan_model("GOGaUModel.stan") 
optimizing(mystanGOGaU, data = $\operatorname{list}(\mathrm{n}=$ length $(\mathrm{x}), \mathrm{x}=\mathrm{x})$

$$
\text { hessian=TRUE) }
$$

sampling(mystanGOGaU, data = $\operatorname{list}(\mathrm{n}=\operatorname{length}(\mathrm{x}), \mathrm{x}=\mathrm{x})$,

$$
\text { iter=10000) }
$$

Program developed in R of optimization for LSE method in subsection 4.2. Other methods are the same.

$\left.1: \mathrm{n} /(\mathrm{n}+1))^{\wedge} 2\right)$

$$
\begin{aligned}
& \mathrm{t}=\operatorname{sort}(\mathrm{x}) \\
& \mathrm{LSE}=\text { function(para) }
\end{aligned}
$$

$$
\operatorname{sum}((\mathrm{pGOGaU}(\mathrm{t}, \quad \text { para }) \quad-
$$

lower $=\mathrm{c}(0.005,0.005)$,

$$
\operatorname{optim}(\operatorname{par}=\mathrm{c}(1,1)
$$

BFGS-B", hessian=TRUE)

\section{Creative Commons Attribution License 4.0 (Attribution 4.0 International, CC BY 4.0)}

This article is published under the terms of the Creative Commons Attribution License 4.0

https://creativecommons.org/licenses/by/4.0/deed.en_US

\section{B Figures}

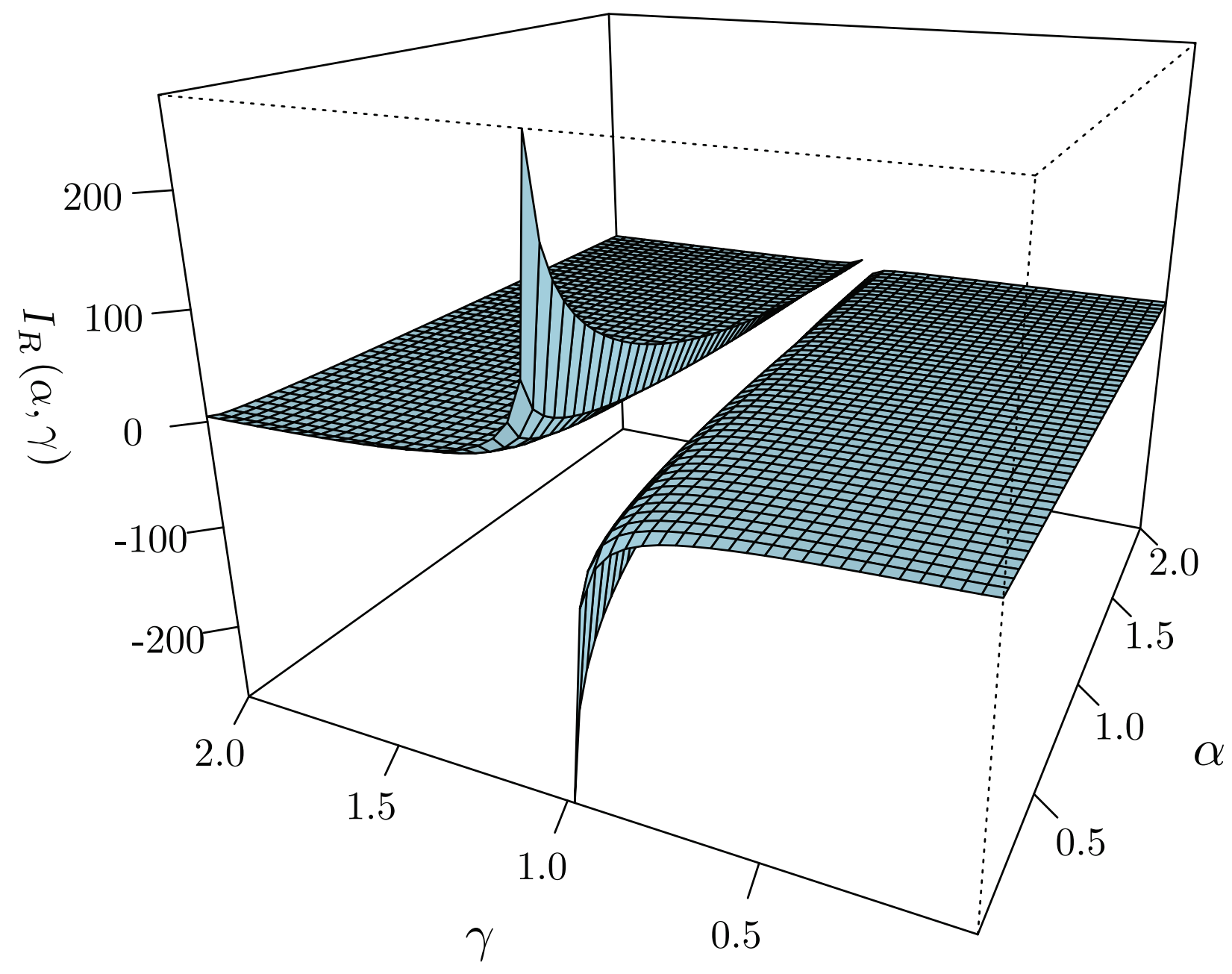




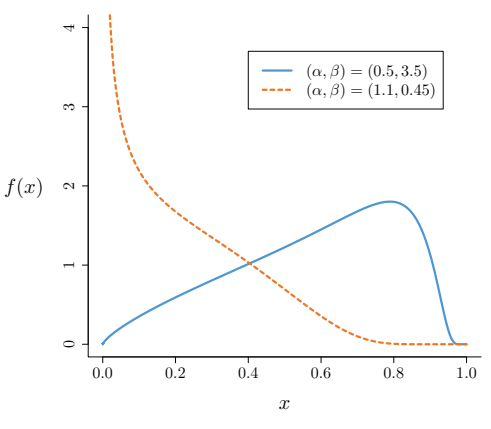

Figure 5: Two density functions for simulation study.
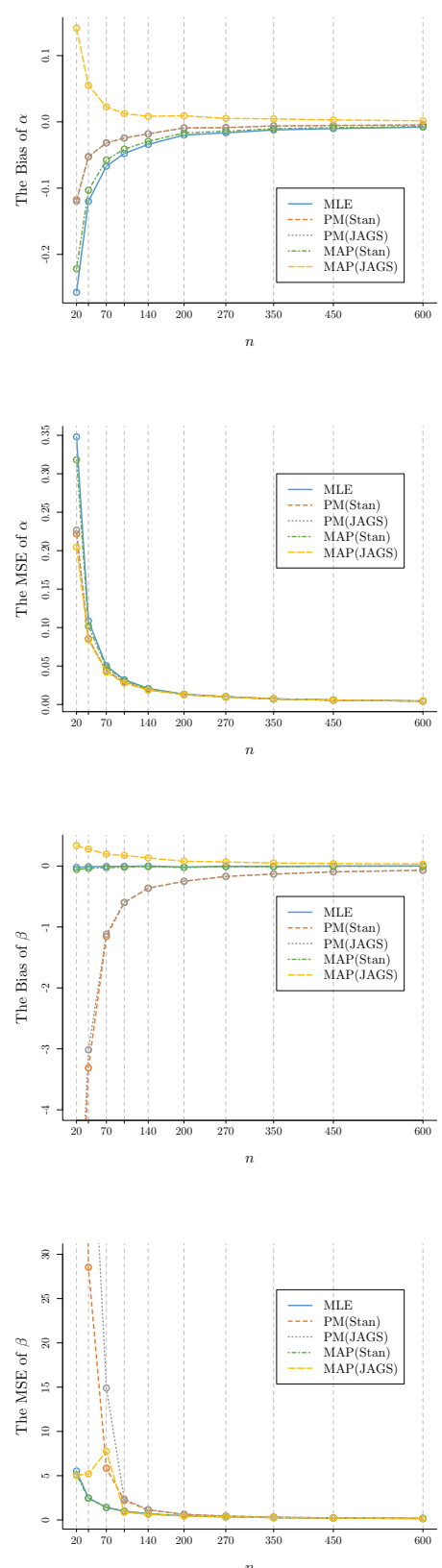

Figure 6: The Biases and MSEs of $\hat{\alpha}$ and $\hat{\beta}$ versus $\mathrm{n}$ when $(\alpha, \beta)=(0.5,3.5)$. 

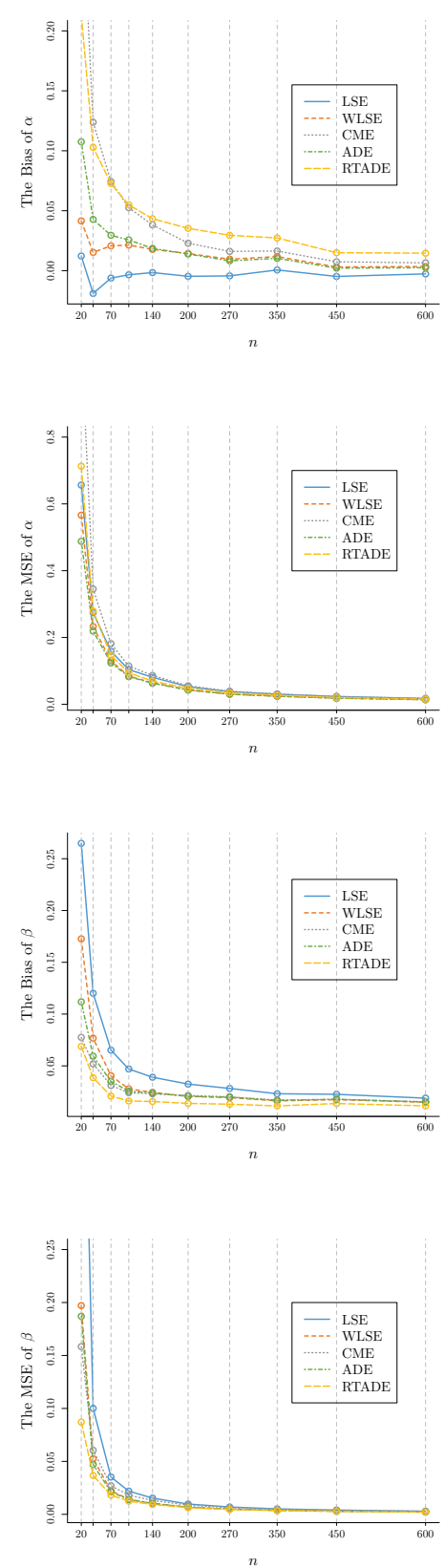

Figure 7: Biases and MSEs of $\hat{\alpha}$ and $\hat{\beta}$ versus $\mathrm{n}$ when $(\alpha, \beta)=(0.5,3.5)$.

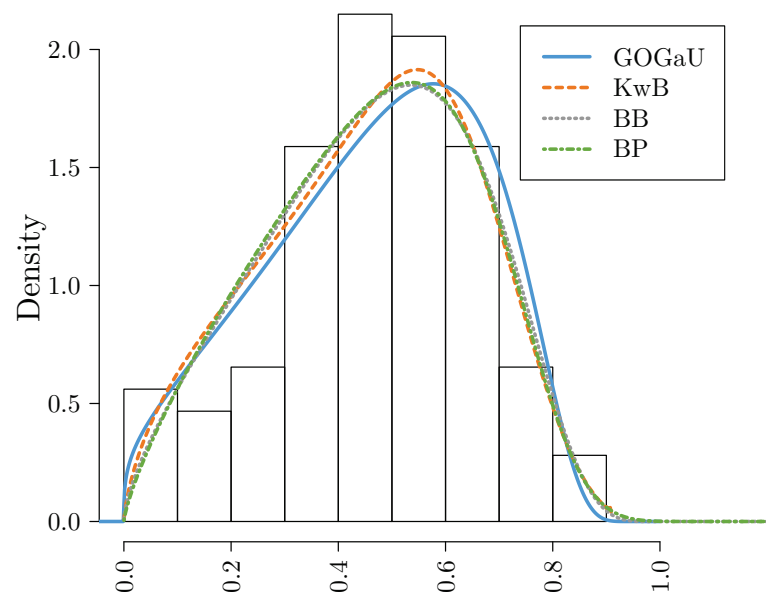

Figure 8: Histogram and estimated pdfs for the total milk production data set.

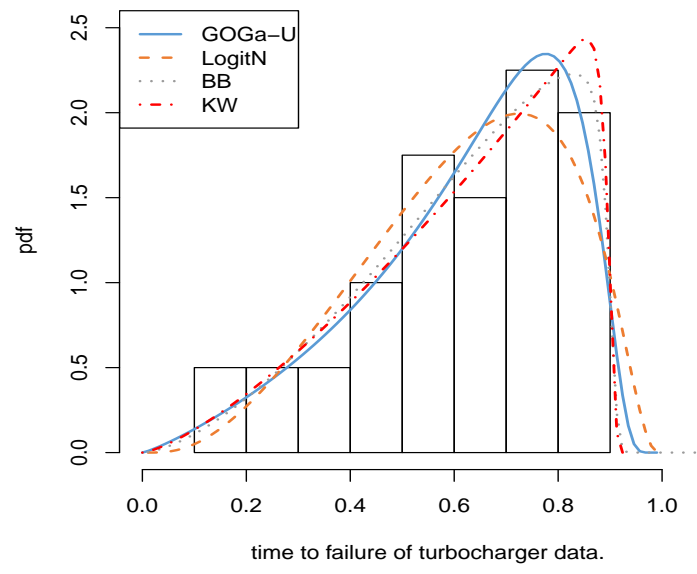

Figure 9: Histogram and estimated pdfs for the time to failure $\left(10^{3} \mathrm{~h}\right)$ of turbocharger data set. 

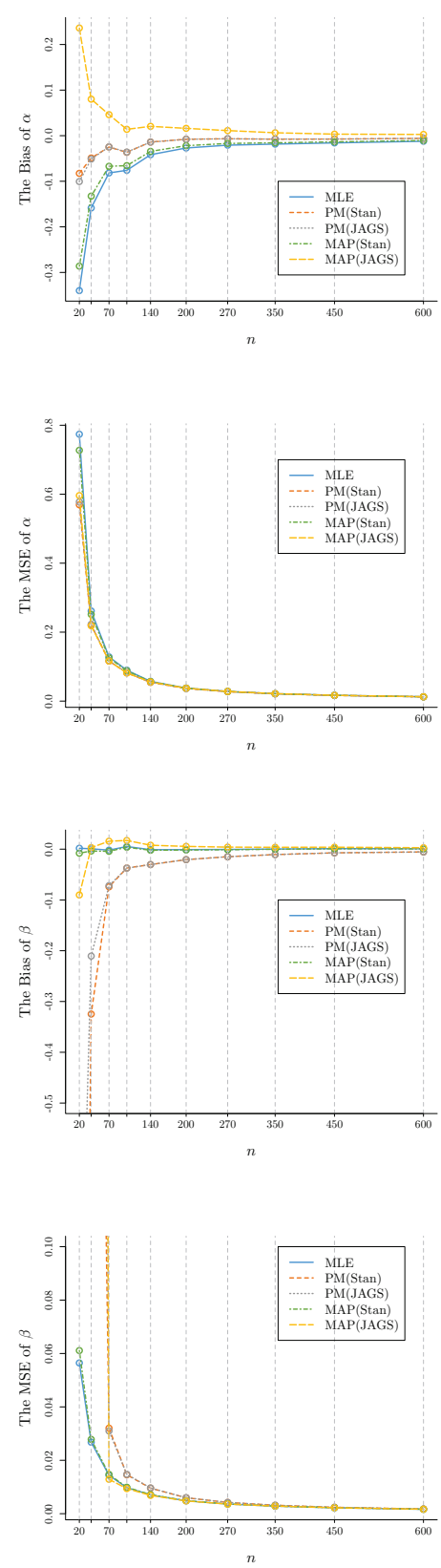

Figure 10: The Biases and MSEs of $\hat{\alpha}$ and $\hat{\beta}$ versus $n$ when $(\alpha, \beta)=(1.1,0.45)$.
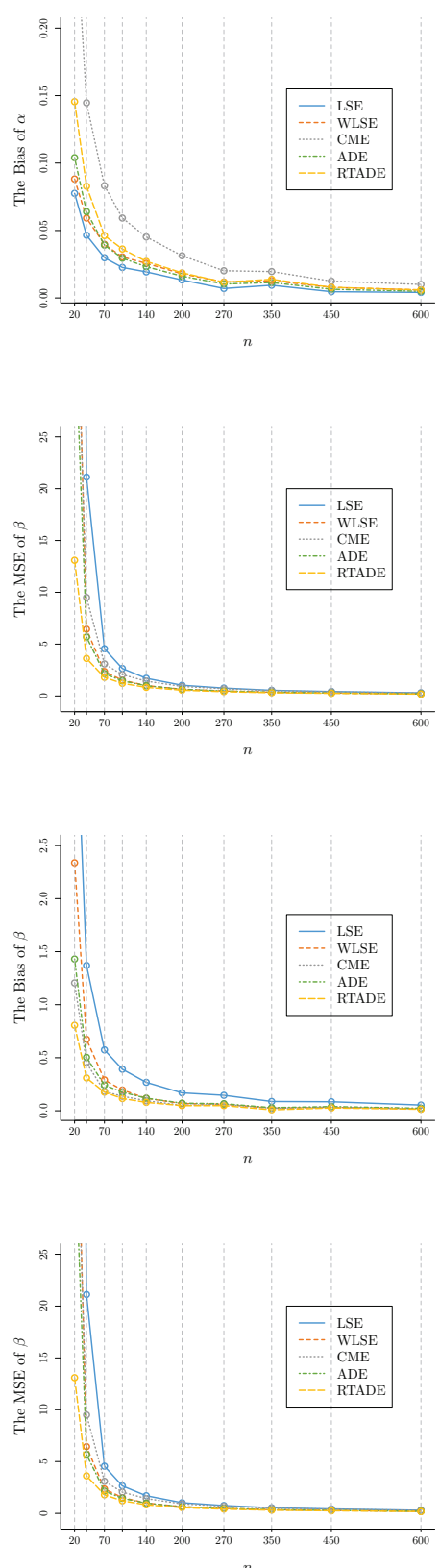

Figure 11: Biases and MSEs of $\hat{\alpha}$ and $\hat{\beta}$ versus $\mathrm{n}$ when $(\alpha, \beta)=(1.1,0.45)$. 